\section{Present and future of siderophore-based therapeutic and diagnostic approaches in infectious diseases}

\author{
Gilda Tonziello, ${ }^{1}$ Emanuela Caraffa, ${ }^{1}$ \\ Biagio Pinchera, ${ }^{2}$ Guido Granata, ${ }^{1}$ \\ Nicola Petrosillo ${ }^{1}$ \\ ${ }^{1}$ National Institute for Infectious Diseases \\ "L. Spallanzani" - IRCCS, Rome; \\ ${ }^{2}$ University of Naples "Federico II", \\ Naples, Italy
}

\begin{abstract}
Iron is an essential micronutrient required for the growth of almost all aerobic organisms; the iron uptake pathway in bacteria therefore represents a possible target for novel antimicrobials, including hybrids between antimicrobials and siderophores. Siderophores are low molecular weight iron chelators that bind to iron and are actively transported inside the cell through specific binding protein complexes. These binding protein complexes are present both in Gram negative bacteria, in their outer and inner membrane, and in Gram positive bacteria in their cytoplasmic membrane. Most bacteria have the ability to produce siderophores in
\end{abstract} order to survive in environments with limited concentrations of free iron, however some bacteria synthetize natural siderophore-antibiotic conjugates that exploit the siderophore-iron uptake pathway to deliver antibiotics into competing bacterial cells and gain a competitive advantage. This approach has been referred to as a Trojan Horse Strategy. To overcome the increasing global problem of antibiotic resistance in Gram negative bacteria, which often have reduced outer membrane permeability, siderophore-antibiotic hybrid conjugates have been synthetized in vitro. Cefiderocol is the first siderophore-antibiotic conjugate that progressed to late stage clinical development so far. In studies on murine models the iron-siderophore uptake pathway has been also exploited for diagnostic imaging of infectious diseases, in which labelled siderophores have been used as specific probes. The aim of this review is to describe the research progress in the field of siderophore-based therapeutic and diagnostic approaches in infectious diseases.

\section{Siderophore-based iron uptake pathways in bacteria}

Iron is essential for the growth of almost all aerobic organisms, except Lactobacilli $^{1}$ and Borrelia burgdorferi. ${ }^{2}$ In mammals iron in circulating blood is largely bound to transferrin and soluble ferrous iron $(\mathrm{Fe} 2+)$ is rapidly oxidized to its insoluble ferric form $(\mathrm{Fe} 3+)$ such that the free iron concentration in the blood is in the region of $10^{-24} \mathrm{M}^{1,3}$ Iron-sulfur groups (Fe-S) are present in heme binding proteins. Moreover, in mammals' ribonucleotide reductions regulate various functions, including heme synthesis, oxygen transport and DNA synthesis. Furthermore, iron homeostasis is related to the inflammatory response. ${ }^{4}$ In fact, during inflammation and/or infection, the host can further reduce the availability of trace minerals in an attempt to limit pathogenicity and decrease nutrient pollution. This process is known as nutritional immunity and is a mechanism by which low circulating levels of iron and zinc can be protective, while higher levels can be associated with increased rates of infection and faster progression of disease. ${ }^{5}$ A large body of data has shown that iron overload is associated with a poor clinical outcome in a series of infectious diseases such as acquired immunodeficiency syndrome, tuberculosis ${ }^{6-}$ ${ }^{9}$ and malaria; ${ }^{10}$ furthermore, dietary supplementation exacerbates the overall mortality rate in endemic areas for these infectious diseases. ${ }^{11-15}$

Control over iron homeostasis therefore represents a central battlefield. The control of iron homeostasis may influence the course of an infectious disease in favour of the mammalian host or the pathogenic invader. Human pathogens such as staphylococci and Salmonella spp., or fungi including Aspergillus spp, have the ability to evade nutritional immunity in order to cause disease. ${ }^{16-19}$ The microbes have also developed strategies to acquire iron from their hosts. One of these strategies includes the acquisition of iron by siderophores. Siderophores are low molecular weight iron chelators (500-1,500 Da) that possess a high affinity for iron and coordinate with the ferric ions through a chemical bond, i.e. the Lewis base. ${ }^{20}$ During nutritional immunity, low iron levels induce transcriptional activation of iron regulators which leads to upregulation of siderophore synthesis and iron regulated outer membrane proteins (iROMPs). Siderophore production therefore increases with decreasing concentration of iron in the environment. ${ }^{21}$ Iron acquisition begins with the binding of siderophores to the available free ferric ions, forming an iron-siderophore complex. Siderophores can be classified into five main classes according to the structure of their iron-binding scaffold: catecholates and phenolates (also termed as "aryl caps"),
Correspondence: Nicola Petrosillo, National Institute for Infectious Diseases "L. Spallanzani" - IRCCS, Via Portuense 292, 00149 Rome, Italy.

Tel.: +39.06.55170260

E-mail: nicola.petrosillo@inmi.it

Key words: iron uptake, siderophores, multidrug-resistant organisms, cefiderocol.

Acknowledgements: the authors would thank Christopher Longshaw for critical reading and correction of the manuscript.

Contributions: the authors contributed equally.

Conflict of interest: NP received fees as speaker/scientific board from MSD, Pfizer, Angelini, Zambon, Johnson \& Johnson, Shionogi, Becton \& Dickinson, Cepheid. GT, EC, BP, GG declare no conflict of interest.

Funding: ricerca Corrente IRCCS.

Received for publication: 15 June 2019

Accepted for publication: 4 July 2019.

This work is licensed under a Creative Commons Attribution-NonCommercial 4.0 International License (CC BY-NC 4.0).

CCopyright: the Author(s), 2019

Licensee PAGEPress, Italy

Infectious Disease Reports 2019; 11:8208

doi:10.4081/idr.2019.8208

hydroxamate (having a hydroxyl group), carboxylates (carboxyl group) and mixed type. ${ }^{22}$

In addition to the cytoplasmic membrane (CM), Gram negative bacterial cells are also surrounded by an external outer membrane (OM) composed of lipopolysaccharide (LPS), which acts as an additional permeability barrier whilst allowing the absorption of essential nutrients. Small molecules cross the OM by passive diffusion through aqueous porin channels, however siderophore-mediated iron absorption requires specialized iROMPs such as FepA, FecA and FhuA, which bind to specific iron-siderophore complexes. ${ }^{23}$ The structure of these receptors consists of twenty-two barrels of antiparallel filaments that contain extracellular rings that interact with the ferric-siderophore complex and an N-terminal hatching domain. ${ }^{24}$ Active uptake through iROMPs is dependent on the TonB complex (TonB, ExbB and ExbD) which provide the energy required to transport the ironsiderophore complex across the OM against a concentration gradient. The ironsiderophore complex enters the periplasmic space and is then bound to a shuttle-acting periplasmic binding protein (PBP). The 
resulting iron-siderophore-PBP complex is then transported through the cytoplasmic membrane by an $\mathrm{ABC}$ transporter into the bacterial cytoplasm. Once in the cytoplasm, iron is released from the siderophores with two mechanisms: one concerns the reduction of $\mathrm{Fe} 3+$ to $\mathrm{Fe} 2+$ by iron reductase and its transfer to various accepting molecules within the cell membrane or inside the cell; the other regards the hydrolysis of the ironsiderophore complex, which requires specific enzymes. The iron-free siderophore is degraded or secreted by the efflux pumps.

Unlike Gram negative bacteria, Gram positive bacteria do not have an external membrane or periplasmic zone. The cell wall of Gram positive bacteria is composed of forty layers of murein and about 30-70\% of the dry mass of the wall is constituted by the peptidoglycan which contains teichoic and lipoteichoic acids. Therefore, they possess a simple uptake mechanism involving a siderophore-binding protein and an associated permease located on the cell membrane. ${ }^{25,26}$ The complex is transported with the $\mathrm{ABC}$ transporter into the cytoplasm of this Gram-positive bacterium.

\section{Siderophore-antibiotic conjugates}

\section{Natural siderophore-antibiotic con- jugates}

In their attempt to gain advantage over other microorganisms and to survive in low iron concentration environment, some bacteria produce natural siderophore-antibiotic conjugates, named sideromycins, with antimicrobial activity against different bacteria species. This strategy to deliver the antibiotic molecule to its site of action through the bacteria membranes by using the specific siderophore uptake pathway has been named the "Trojan Horse Strategy". 27,28

An example of a natural sideromycin is albomycin, a compound discovered in 1947 and produced by some Streptomyces strains with antimicrobial activity against several Gram positive and Gram negative bacteria. ${ }^{29}$ Albomycin is made of a trihydroxamate siderophore linked by a serine spacer to a thionucleoside moiety that inhibits aminoacyl tRNA synthetase. Albomycin gains access to the bacteria cytoplasm through a specific active siderophore transmembrane transport pathway; once inside the cytoplasm a serine protease cleaves the thionucleoside moiety from the siderophore, allowing the former to stop the protein synthesis by blocking the aminoacyl tRNA synthetase. ${ }^{26}$

Albomycin is active against
Enterobacteriaceae, except Proteus and Morganella, and against some Gram positive bacteria, including $S$. aureus and $S$. pneumoniae. Albomycin has been shown to be effective in treating infections from $S$. pneumonia and $Y$. enterocolitica in murine infection models. ${ }^{28}$ Albomycin resistant bacteria can be selected both in vitro and in vivo, however when exposed to albomycin these strains show a reduced fitness in the mouse infection model compared with wild type parent strains. ${ }^{28}$

Even though albomycin had been experimented to treat infections in humans in the ' $50 \mathrm{~s},{ }^{30}$ it was subsequently abandoned for many years, due to difficulties in synthetizing enough molecules for commercial purpose. New interest in this old antibiotic has been increasing in the last years and in 2018 Lin et al. chemically synthetized albomycin in vitro. ${ }^{31}$

Another example of a natural sideromycin is salmycin, isolated from Streptomyces violaceus in $1995 .{ }^{32}$ Its structure is made of a trihydroxamate siderophore, known as danoxamine, linked by a succinoyl spacer with an aminoglycoside antibiotic. As for albomycin, salmycin gains access through hydroxamate siderophore membrane transport pathway to the cytoplasm where the aminoglycoside pharmacophore can act by inhibiting protein synthesis. Salmycin has activity primarily against Gram positive bacteria, namely staphylococci and streptococci, while it is not effective against most Gram negative bacteria. Salmycin was tested in vivo in mice models infected with $S$. aureus, where it was shown to rapidly loose its activity, due to its instability. ${ }^{32,33}$

Escherichia coli produces several different catecholate type siderophoremicrocin conjugates that inhibit the growth of other Gram negative Enterobacteriacae. These molecules have different mechanisms of action but use the same "Trojan horse" system to gain access to cytoplasm through the catecholate siderophore transport proteins in the outer and inner membranes of Enterobacteracae. Microcins are ribosomally synthetized peptides that are post translationally modified with the attachment of a catecholate siderophore moiety and whose transcription is induced by low iron environment concentration through the Fur transcription regulator protein. ${ }^{33}$

In 2016 Nakamura et al..$^{34}$ identified a new antifungal agent named ASP2397, whose structure is similar to ferrichrome, a low-molecular-weight siderophore. ASP2397 is produced by a fungal strain isolated from Malaysian leaf litter, belonging to Acremonium species. This compound and its derivatives are in vitro inhibitors of $A$ fumigatus growth.

\section{Synthetic siderophore-antibiotic conjugates}

Researchers have been inspired by natural sideromycins and the "Trojan horse" strategy to overcome the problem of multidrug resistant (MDR) Gram negative bacteria by synthetizing new siderophoreantibiotic conjugates in vitro. ${ }^{35}$

Unfortunately, nowadays clinicians do not have many weapons to fight against MDR Gram negative organisms such as Acinetobacter

baumannii, Enterobacteriaceae and Pseudomonas aeruginosa which are responsible for healthcare-associated infections with high morbidity and mortality. ${ }^{36}$ Under the pressure of antibiotic overuse, these bacteria developed several mechanisms of resistance to the existing antibiotics, including alteration of membrane permeability, synthesis of enzymes that inactivate antibiotics (i.e. beta-lactamases), modifications in antibiotic target binding sites and expression of efflux channels that actively pump the antibiotic outside the target cell. ${ }^{37}$

One of the most important challenges in the discovery of new drugs active against Gram negative organisms is the need to overcome the obstacle of low permeability of the outer membrane so that the antibiotic could gain access to its target binding site. One novel approach to achieve this goal is the use of siderophore-antibiotic conjugates, that exploit the binding of ferricsiderophore complex to its outer membrane receptor and its active transport through outer and inner membrane to gain access to periplasmic space and cytoplasm. ${ }^{38}$

Even though antibiotics with virtually every mechanism of action might be used to exploit the Trojan horse strategy, to date beta-lactam antibiotics have been the starting point for the in vitro synthesis of new artificial antibiotic-siderophore conjugates, as their cell wall target is located in the periplasmic space and only requires transport across one bacterial membrane.

Following, we report some of the artificial antibiotic-siderophore conjugates that were synthetized and tested in the past years; among them, only one, cefiderocol, has succeeded in reaching late stage clinical development and phase III clinical trials.

\section{Siderophore-antibiotic conju- gates containing beta lactam antibiotics}

In 1987 Watanabe et al. ${ }^{39}$ synthesized a 
novel siderophore-cephalosporin conjugate, named E-0702, with antimicrobial activity against Gram negative bacteria, including $K$. pneumonia, S. Typhimurium, $S$. marcescens, $P$. mirabilis and $P$. aeruginosa. Resistant bacteria spontaneously developed, but resistance was not due to increased expression of beta-lactamase, nor to reduction or loss of $\mathrm{OmpF}$ and $\mathrm{OmpC}$ porin proteins in the outer membrane; resistance was associated with a mutation in ton $B$ gene that, as previously mentioned, plays a pivotal role in the siderophoremediated iron uptake. They also demonstrated that E-0702 had the most effective antibacterial activity on iron starved bacteria, while it was ineffective in iron supplemented bacteria; so they hypothesized that E-0702 was incorporated into bacteria through the tonB dependent siderophoreiron transport system.

The siderophore-iron mediated uptake pathway was more deeply investigated by Curtis et al. in $1988 .{ }^{40}$ These authors tested in vitro synthetized C-3' catechol-substituted aminothiazolyl-oxime cephalosporins against $E$. coli mutant strains that did not express specific outer membrane receptors of the tonB-dependent pathway, with the aim to identify which proteins were involved in the uptake and antibacterial activity of these siderophore-antibiotic conjugates and therefore the possible mechanisms of resistance that could jeopardize their activity.

They found that "Fiu" and "Cir" outer membrane proteins were involved in the cathecol-siderophore-antibiotic conjugates transport and that bacteria not expressing fiu and cir genes had a reduced susceptibility to the compounds.

In 1992 Brochu et al. ${ }^{41}$ tested the in vitro antimicrobial activity of two synthetic carbacephalosporin-siderophore conjugates against E. coli. The siderophore components of these two conjugates were a hydroxamic acid-based and a catecholbased scaffolds, respectively. ${ }^{41}$ These two carbacephalosporin-siderophore conjugates bound different outer membrane receptor proteins, FhuA and Cir receptors, respectively. In vitro studies suggested that bacteria with a mutated outer membrane receptor for hydroxamate (FhuA) or catecholate (Cir) based siderophores were rapidly selected; these organisms were resistant to that specific antibiotic conjugate containing the hydroxamate or catecholate based siderophore mojetiy but they were still susceptible to the antimicrobial activity of the other conjugate. So, the researchers combined both the iron binding hydroxamic acid and catechol moieties in a new mixed ligand siderophore conjugate that was able to alternatively bind both the outer membrane receptors, obtaining a greater inhibitory activity concomitantly with a lower frequency of resistance.

Many other siderophore-beta lactams conjugates with antipseudomonal activity were synthetized and studied in vitro. Among these, the catechol-conjugated cephalosporin (GR69153) synthetized by Silley et al. in $1990^{42}$ was active in vitro against E. coli and $P$. aeruginosa, with a lower minimal inhibitory concentrations (MIC) observed in iron depleted medium; Chin et $a l .{ }^{43}$ demonstrated that this compound was active also against other Enterobacteriaceae and Gram positive bacteria. Wittmann et al. ${ }^{44}$ in 2002 synthetized and tested acylated bis- or triscatecholates and mixed biscatecholate hydroxamates conjugates with ampicillin, amoxicillin and cefaclor. These compounds were highly active in vitro against Gram-negative bacteria, especially P. aeruginosa and S. maltophilia.

Ji et al. in 2012 synthetized two artificial tris-catecholate siderophore conjugates with ampicillin and amoxicillin that were active against $P$. aeruginosa..$^{45}$ These Gram negative bacteria, although having the periplasmic binding proteins (PBP) targeted by these beta-lactams antibiotics, are actually resistant to them because of their impairment to pass through the outer membrane porins and reach the periplasmic space. These authors demonstrated that both these beta-lactams siderophore conjugates were active against $P$. aeruginosa and that their antibacterial activity was affected by iron concentration, as it was higher in low iron concentration environment.

In the last years, new interest in siderophore-antibiotic conjugates raised because of the increased spread of MDR Gram negative bacteria. Recently, new siderophore-beta-lactams compounds active against Gram negative bacteria have been synthetized. Flanagan et al. synthetized a series of siderophore-conjugated monocarbam antibiotics, one of which is MC-1, active in vitro against MDR $P$. aeruginosa, ESBL-producing members of the Enterobacteriaceae and Acinetobacter baumannii. ${ }^{46}$ Anyway further investigation is needed to overcome the hydrolytic instability of this compound. In murine pulmonary infection model, the high affinity of these compounds to the plasma proteins limited their effectiveness. ${ }^{46}$

A monosulfactam conjugated with an iron-chelating dihydroxypyridone moiety (BAL30072) that is active against MDR $P$. aeruginosa and Acinetobacter sp. isolates, including many carbapenem-resistant strains has recently been discovered. ${ }^{47}$ This molecule was also tested in combination with imipenem, meropenem, and doripenem against selected strains of Enterobacteriaceae, $P$. aeruginosa, and $A$. baumannii, demonstrating synergistic effects in most species except $A$. baumannii. In clinical trials with BAL30072 elevations of liver enzymes have been observed.

Cefiderocol (S-649266) is the first siderophore-antibiotic conjugate that has progressed to phase III clinical trials, so far. $^{48}$ It is a catecholate-siderophorecephalosporin conjugate, which has structural features from the $3^{\text {rd }}$ and $4^{\text {th }}$ generation cephalosporins, ceftazidime and cefepime, combined with a catechol moiety at the C3position which enables it to bind to ferric iron and to be taken into the cell via siderophore transporter proteins. Cefiderocol has in vitro and in vivo activity against several Gram negative organisms, including carbapenem-resistant Enterobacteriaceae, P. aeruginosa, and $A$. baumannii. Its high activity against Gram negative bacteria is due to the Trojan horse delivery strategy and to its high stability to beta-lactamases inactivation. In fact, Cefiderocol is active against OXA-58, NDM- and IMP-producing A. baumannii isolates, $P$. aeruginosa isolates and carbapenem-resistant Enterobacteriaceae, including KPC-, NDM-, IMP-, VIM-producing isolates. ${ }^{49}$ Clinical trials have already confirmed the ability of Cefiderocol to treat infections caused by common Gram negative pathogens. In a prospective, multicenter, double-blind, non-inferiority trial, Cefiderocol $2000 \mathrm{mg}$ three times daily was superior to imipenem-cilastatin $1000 \mathrm{mg}$ three times daily for the treatment of complicated urinary tract infections. ${ }^{50}$ The study enrolled 452 hospitalized adult patients including immunocompromised patients. Patients received Cefiderocol or imipenem/cilastatin for 7-14 days. At test of cure, the primary efficacy endpoint was achieved by $183(73 \%)$ of 252 patients in the Cefiderocol group and $65(55 \%)$ of 119 patients in the imipenem-cilastatin group, establishing the non-inferiority of Cefiderocol..$^{50}$ Two other phase III studies on Cefiderocol were conducted by Shionogi \& Co., Ltd., Japan. ${ }^{51,52}$ One of these evaluates the efficacy of Cefiderocol in the treatment of serious infections (urinary tract infections, pneumonia, blood stream infections $)^{51}$ caused by carbapenem-resistant Gram-negative pathogens and the other compares the drug with meropenem for the treatment of hospital-acquired bacterial pneumonia and ventilation-associated pneumonia. ${ }^{52}$ Cefiderocol seems to be well tolerated, with a profile of side effects similar to other cephalosporins, including gastrointestinal 
symptoms and phlebitis. GT-1 is another siderophore conjugated cephalosporin, discovered by LegoChem Biosciences in $2017 .{ }^{53}$ It is active against MDR $P$. aeruginosa and $A$. baumannii in vitro and in murine infection model. Its association with a new beta-lactamase inhibitor GT-055 widens its activity also against carbapenem producing Enterobacteriaceae, including Class A and some B, C and D beta-lactamases producers.

\section{Siderophore-antibiotic conjugates containing non-beta-lactam antibiotics}

In 1987 Pugsley et al.$^{54}$ tested in vitro a semisynthetic siderophore-rifamycin conjugate CGP 4832 finding that its activity was comparable to rifampicin against Gram positive bacteria but that it was $80 \sim 400$ times more potent than rifampicin against certain Gram negative species. This compound was actively transported across the outer membrane of Gram negative bacteria through the siderophore specific transporter FhuA, reaching high concentration in the periplasmic space and passively diffusing across the cytoplasmic membrane to the cytoplasm, where the rifamycin could bind to its target, the RNA polymerase. ${ }^{55}$

Wencewicz et al. ${ }^{56}$ in 2013 synthetized a new hydroxamate-siderophore-fluorochinolone conjugate using ciprofloxacin, that had a narrower spectrum of action than ciprofloxacin alone, as it was selectively active against Gram positive bacteria. The authors hypothesized that this compound exploited the specific active siderophore uptake pathway expressed on the cytoplasmic membrane of $S$. aureus to reach its cytoplasmic biological target.

Recently Gosh et al. in 2018 synthetized an artificial siderophore-daptomycin conjugate, using a mixed ligand ana$\log$ of the natural Acinetobacter baumannii selective siderophore, fimsbactin. This conjugate was selectively active against $A$. baumannii but it was not active against Pseudomonas aeruginosa, Burkholderia and $E$. coli, and retained activity against $S$. aureus, even though lower than daptomycin alone..$^{57}$

\section{Iron-siderophore uptake pathway as a target for antimicrobial drugs against Mycobacterium tuberculosis}

Iron-siderophore uptake pathway has also been studied as a target for antimicrobial drugs, particularly against $M$. tuberculosis. ${ }^{58,59} \mathrm{In}$ fact, as for other bacteria, in $M$. tuberculosis the iron transport inside the cell is mediated by siderophores, named desferricarboxymycobactin (CMb) and mycobactin $(\mathrm{Mb})$. The former is produced upon iron shortage and is excreted outside the cell where it binds to $\mathrm{Fe} 3+$, present as insoluble or protein-bound iron, and forms ferricarboxymycobactin. The transfer of $\mathrm{Fe} 3+$ from ferricarboxymycobactin to mycobactin $(\mathrm{Mb})$, located on the cytoplasmic membrane, is mediated by a shuttle protein; subsequently mycobactin transfers iron to a transmembrane iron transporter that catalyzes the reduction of $\mathrm{Fe} 3+$ to $\mathrm{Fe} 2+$ and mediates the ATP-dependent transfer of iron into the cytoplasm, where the iron is partly used for metabolic processes, partly bond to storage proteins. ${ }^{58}$

Several molecules with anti-tubercular activity that are active on the iron siderophore uptake pathway have been studied. ${ }^{59}$ Among them, pyrazolopyrimidinone is a ferrous ion chelating agent, that inhibits the use of iron by the cell even in presence of increased levels of iron; the 3,5-diaryl pyrazoline (DAP) derivatives and 2(E)-2-benz y lidene-N-hydrox y hydrazine carbo(Ox/Thio/Oximid)amide (BHHC) derivatives contain an hydroxyphenyl moiety similar to that of siderophores, with a higher iron binding affinity and compete with the siderophore for the iron binding, interfering with the siderophore mediated iron transport. Also, some natural siderophores synthetized by other organisms have shown anti-tubercular activity: one is extracted from roots of Tephrosia purpurea, the other is named transvalencin $\mathrm{Z}$ and is obtained from Nocardia transvalensis. Other researchers are focusing on the steps of siderophores biosynthesis pathway and their excretion from the cell, mediated by the trans-membrane protein complex, as targets of new compounds that can interfere with the siderophore-iron uptake and inhibit the microorganism growth. ${ }^{59}$

\section{Siderophore-based probes: new approach in the development of diagnostic systems}

In the diagnostic field siderophores have been studied as a tool for molecular imaging for fungal infections. Labelled siderophores can be prepared either by replacing Fe with the appropriate radiometal in the natural iron-siderophore complex or artificially by modifying the natural siderophore with the addition of a chromophore suitable for optical imaging. ${ }^{60}$ The radiologic or optical images obtained with these new pathogen-specific probes of infection can be combined with the other radiological and nuclear imaging techniques to gain a more specific and aetiological diagnosis. ${ }^{61}$

In fact, radiological imaging techniques such as computed tomography, magnetic resonance imaging and ultrasonography, widely used in clinical practice for identification of infection, have major limitations in specificity. By contrast, nuclear imaging techniques including Positron Emission Tomography (PET) and Single Photon Emission Computed Tomography (SPECT) have a rich history of different radiolabelled probes (radiopharmaceuticals) for imaging of infectious processes in patients, such as $111 \mathrm{In}-$ or $99 \mathrm{mTc}-$ labelled leucocytes, 99mTc-anti-granulocyte antibody, $99 \mathrm{mTc}$ diphosphonates in the context of bone scanning, 67Ga-citrate and 2-[18F]-fluorodeoxyglucose. Anyway, these probes target predominantly secondary effects of infection such as increased blood flow and vascular permeability, activated endothelial cells or polymorphonuclear cell migration at site of infection.

Siderophore-iron transporters-mediated iron uptake is universally conserved in the fungal kingdom, even in species not producing siderophores such as Saccharomyces cerevisiae, Candida spp. and Cryptococcus neoformans. ${ }^{62,63}$ Moreover, there is evidence that the iron-siderophore uptake system is active during infection; siderophore biosynthesis and uptake are transcriptionally upregulated during iron starvation in vitro as well as in vivo in a murine model for pulmonary infection with the mold Aspergillus fumigatus, and siderophoremediated iron assimilation plays a major role for its virulence. ${ }^{64}$ In particular, the siderophore of Aspergillus fumigatus, triacetylfusarinine $\mathrm{C}$ (TAFC) was shown to be able to extract iron from host transferrin. ${ }^{65}$ Similarly, siderophore biosynthesis was shown to be crucial for the virulence of numerous bacterial species including, Yersinia pestis, Mycobacterium tuberculosis or Pseudomonas aeruginosa. ${ }^{66}$

Aspergillus fumigatus uses two high affinity iron uptake systems: reductive iron assimilation (Fe2+ specific) and siderophore-assisted iron acquisition ( $\mathrm{Fe} 3+$ specific), but only the latter system is essential for virulence of $A$. fumigatus.

Aspergillus fumigatus produces two hydroxamate-type siderophores, namely fusarinine $\mathrm{C}$ (FSC) and its $\mathrm{N}$-acetylated derivative triacetylfusarinine $\mathrm{C}$ (TAFC), for extracellular iron acquisition. During infection, A. fumigatus faces an iron-starvation environment and excretes siderophores for stealing host iron. After chelation of iron, siderophore-iron complexes are taken up 
through siderophore-iron transporters, which are members of a subfamily of major facilitator protein superfamily.

In A. fumigatus, MirB has been identified as a specific transporter for TAFC whereas the FSC transporter still remains to be identified. The MirB transporter is highly upregulated during infection and is found in A. fumigatus, but not in bacteria and several other fungal strains. ${ }^{66}$ Furthermore, humans lack siderophore-iron transporters such as MirB and therefore specific substrates do not interact with human cellular systems and the siderophore triacetylfusarinine C (TAFC) for iron acquisition is essential for the virulence of Aspergillus. Therefore, TAFC is a specific marker for invasive aspergillosis.

The possibility of using a $68 \mathrm{Ga}$ siderophore as an Aspergillus-specific probe in PET imaging for diagnosis of Aspergillus fumigatus infection was investigated by labelling this desferrisiderophore. It was shown that positron emission tomography (PET) imaging with [68Ga]TAFC exhibited excellent targeting properties in an $A$. fumigatus rat infection model.67,68 High contrast imaging of Aspergillus fumigatus pulmonary infection in a rat model was achieved using micro-PET/CT technology, exhibiting pronounced accumulation of $68 \mathrm{Ga}-\mathrm{TAFC}$ in infected areas extremely early after onset of infection, which increased with severity of infection and correlated with abnormal CT images. Significant accumulation of $68 \mathrm{Ga}$ TAFC was found neither in sterile inflammations nor in tumour cells, which also have a high iron metabolism.

It was also investigated the uptake of $68 \mathrm{GaTAFC}$ in a number of different fungal and bacterial species, which revealed high specificity for Aspergillus species, with no significant uptake by Candida and bacterial species, in particular. On the basis of these results on murine model, 68Ga-labelled siderophores, in particular 68Ga-TAFC, might have a high potential to be used as radiopharmaceuticals to specifically image Aspergillus infections in patients. ${ }^{65}$

Overall, radiolabelled siderophores have the potential to be a highly specific tool for infection imaging, considering the essential role of the siderophore system for iron acquisition and virulence of pathogens together with its upregulation during infection, and considering that these systems are not utilized by mammals. Selecting appropriate siderophores can also provide a high specificity for particular microorganisms, distinguishing between fungal and bacterial infections.

\section{Conclusions}

The siderophore-drug complex represents today a new and innovative therapeutic strategy against multidrug resistant (MDR) Gram-negative strains such as Acinetobacter

baumannii,

Enterobacteriaceae and Pseudomonas aeruginosa. Therefore, there is a growing interest in these innovative molecules. The Trojan horse strategy is a way to overcome the low permeability of Gram-negative outer membrane, exploiting a nutrientuptake pathway to transport the antibiotic inside the periplasmic space. Inspired by the antibiotic properties of natural syderomicins, several researchers and pharmaceutical companies have developed siderophore-antibiotic hybrids. Up to date the catecholate-siderophore-cephalosporin conjugate S-649266, also called cefidero$\mathrm{col}$, is the first compound of this class that has progressed to clinical trials. Siderophores have also a large number of clinical applications. Iron-siderophore uptake pathway has also been studied as a target for other antimicrobial drugs, particularly against $M$. tuberculosis. Moreover, in the diagnostic field siderophores have been studied as a tool for molecular imaging for fungal infections.

\section{References}

1. Pandey A, Bringel F, Meyer JM. Iron requirement and search for siderophores in lactic acid bacteria. Appl Microbiol Biotechnol 1994;40:735-9.

2. Posey JE, Gherardini FC. Lack of a role for iron in the Lyme disease pathogen. Science 2000;288:1651-3.

3. Raymond K, Dertz E, Kim S. Enterobactin: an archetype for microbial iron transport. Proc Natl Acad Sci 2003; 100:3584-88.

4. Ganz T, Nemeth E. Iron homeostasis in host defence and inflammation. Nat Rev Immunol 2015;15:500-10.

5. Hennigarn SR, McClung JP. Nutritional Immunity Starving Pathogens of Trace Minerals. Am J Lifestyle Med 2016;10:170-3.

6. Kochan I. The role of iron in bacterial infections, with special consideration of host-tubercle bacillus interaction. Curr Top Microbiol Immunol 1973;60:1-30 .

7. Lounis N, Truffot-Pernot C, Grosset J, et al. Iron and Mycobacterium tuberculosis infection. J Clin Virol 2001;20: 123-6.

8. Murray MJ, Murray AB, Murray MB, et al. The adverse effect of iron repletion on the course of certain infections. $\mathrm{Br}$ Med J 1978;2:1113-5.

9. Ratledge C. Iron, mycobacteria and tuberculosis. Tuberculosis 2004;84: 110-30.

10. Kabyemela ER, Fried M, Kurtis JD, et al. Decreased susceptibility to Plasmodium falciparum infection in pregnant women with iron deficiency. J Infect Dis 2008;198:163-6.

11. Esan MO, van Hensbroek MB, Nkhoma E, et al. Iron supplementation in HIVinfected Malawian children with anemia: a double-blind, randomized, controlled trial. Clin Infect Dis 2013;57: 1626-34.

12. Prentice AM, Verhoef $\mathrm{H}$, Cerami C. Iron fortification and malaria risk in children. JAMA 2013;310:914-5.

13. Zlotkin S, Newton S, Aimone AM, et al. Effect of iron fortification on malaria incidence in infants and young children in Ghana: a randomized trial. JAMA 2013;310:938-47.

14. Sazawal S, Black RE, Ramsan M, et al. Effects of routine prophylactic supplementation with iron and folic acid on admission to hospital and mortality in preschool children in a high malaria transmission setting: community-based, randomised, placebo-controlled trial. Lancet 2006;367:133-43.

15. Soofi S, Cousens S, Iqbal SP, et al. Effect of provision of daily zinc and iron with several micronutrients on growth and morbidity among young children in Pakistan: a cluster-randomised trial. Lancet 2013;382:29-40.

16. Nairz M, Haschka D, Demetz E, et al. Iron at the interface of immunity and infection. Front Pharmacol 2014;5:152.

17. Cassat JE, Skaar EP. Iron in infection and immunity. Cell Host Microbe 2013;13:509-19.

18. Andrews-Polymenis HL, Baumler AJ, McCormick BA, et al. Taming the elephant: salmonella biology, pathogenesis, and prevention. Infect Immun 2010;78:2356-69.

19. Schrettl M, Bignell E, Kragl C, et al. Siderophore biosynthesis but not reductive iron assimilation is essential for Aspergillus fumigatus virulence. J Exp Med 2004;200:1213-9.

20. Boda S, Pandit S, Garai A, et al. Bacterial siderophore mimicking iron complexes as DNA targeting antimicrobials. RSC Adv 2016;6:39245-60.

21. Singh A, Kaushik MS, Srivastava M, et al. Siderophore mediated attenuation of cadmium toxicity by paddy field cyanobacterium Anabaena oryzae. Algal Res 2009;16:63-8.

22. Dhusia K, Bajpai A, Ramteke PW. 
Overcoming antibiotic resistance: Is siderophore Trojan horse conjugation an answer to evolving resistance in microbial pathogens? J Control Release 2018;269:63-87.

23. Köster W. ABC transporter-mediated uptake of iron, siderophores, heme and vitamin B12. Res Microbiol 2001;152:291-301.

24. Krewulak KD, Vogel HJ. Structural biology of bacterial iron uptake. Biochim Biophys Acta 2008;1778:1781-804.

25. Wilson BR, Bogdan AR, Miyazawa M, et al. Siderophores in iron metabolism: from mechanism to therapy potential. Trends Mol Med 2016;22:1077-90.

26. Ballouche M, Cornelis P, Baysse C. Iron metabolism: a promising target for antibacterial strategies. Recent Pat Antiinfect Drug Discov 2009;4:190205.

27. Möllmann U, Heinisch L, Bauernfeind A, et al. Siderophores as drug delivery agents: application of the "Trojan Horse" strategy. Biometals 2009;2:61524.

28. Braun V, Pramanik A, Gwinner T, et al. Sideromycins: tools and antibiotics. Biometals 2009;22:3-13.

29. Reynolds DM, Schatz A, Waksman SA. Grisein, a new antibiotic produced by a strain of Streptomyces griseus. Proc Soc Exptl Biol Med 1947;64:50-4.

30. Guase GF. Recent studies on albomycin, a new antibiotic. Brit Med J 1955;2:1177-9.

31. Lin Z, Xu X, Zhao S, et al. Total synthesis and antimicrobial evaluation of natural albomycins against clinical pathogens. Nat Commun 2018;9:3445.

32. Page MG. Siderophore conjugates. Ann N Y Acad Sci 2013;1277:115-26.

33. Vassiliadis G, Destoumieux-Garzón D, Lombard $\mathrm{C}$, et al. Isolation and characterization of two members of the siderophore-microcin family, microcins $\mathrm{M}$ and H47. Antimicrob Agents Chemother 2010;54:288-97.

34. Nakamura I, Yoshimura S, Masaki T, et al. ASP2397: a novel antifungal agent produced by Acremonium persicinum MF-347833. J Antibiot 2017;70:45-51.

35. Górska A, Sloderbach A, Marszałł MP. Siderophore-drug complexes: potential medicinal applications of the 'Trojan horse' strategy. Trends Pharmacol Sci 2014;35:442-9.

36. O'Neill J. Tackling drug-resistant infections globally: final report and recommendations. 2016. Available from: https://amr-review.org/sites/default/ files/160518_Final\%20paper_with $\% 20$ cover.pdf. Accessed 27/05/2019
37. Ruppé E, Woerther PL, Barbier F. Mechanisms of antimicrobial resistance in Gram-negative bacilli. Ann Intensive Care 2015;5:21.

38. Tillotson GS. Trojan horse antibiotics a novel way to circumvent Gram-negative bacterial resistance? 2016;9:45-52.

39. Watanabe NA, Nagasu T, Katsu K, et al. E-0702, a new cephalosporin, is incorporated into Escherichia coli cells via the tonB-dependent iron transport system. Antimicrob Agents Chemother 1987;31:497-504.

40. Curtis NA, Eisenstadt RL, East SJ, et al. Iron-regulated outer membrane proteins of Escherichia coli K-12 and mechanism of action of catechol-substituted cephalosporins. Antimicrob Agents Chemother 1988;32:1879-86.

41. Brochu A, Brochu N, Nicas TI, et al. Modes of action and inhibitory activities of new siderophore-beta-lactam conjugates that use specific iron uptake pathways for entry into bacteria. Antimicrob Agents Chemother 1992; 36:2166-75.

42. Silley P, JW, Monsey D, et al. Mode of action of GR69153, a novel catecholsubstituted cephalosporin, and its interaction with the tonB-dependent iron transport system. Antimicrob Agents Chemother 1990;34:1806-8.

43. Chin NX, Gu JW, Fang W, et al. In vitro activity and beta-lactamase stability of GR69153, a new long-acting cephalosporin. Antimicrob Agents Chemother 1991;35:259-66.

44. Wittmann S, Schnabelrauch M, Scherlitz-Hofmann I, et al. New synthetic siderophores and their beta-lactam conjugates based on diamino acids and dipeptides. Bioorg Med Chem 2002;10:1659-70.

45. Ji C, Miller PA, Miller MJ. Iron transport-mediated drug delivery: practical syntheses and in vitro antibacterial studies of tris-catecholate sidero-phoreaminopenicillin conjugates reveals selectively potent anti- Pseudomonal activity. J Am Chem Soc 2012; 134:9898-901.

46. Flanagan ME, Brickner SJ, Lall M, et al. Preparation, gram-negative antibacterial activity, and hydrolytic stability of novel siderophore-conjugated monocarbam diols. ACS Med Chem Lett 2011;2:385-90.

47. Page MG, Dantier C, Desarbre E. In vitro properties of BAL30072, a novel siderophore sulfactam with activity against multiresistant gram-negative bacilli. Antimicrob Agents Chemother 2010;54:2291-302.

48. Saisho Y, Katsube T, White S, et al.
Pharmacokinetics, safety, and tolerability of cefiderocol, a novel siderophore cephalosporin for Gram-negative bacteria, in healthy subjects. Antimicrob Agents Chemother 2018;62:e02163-17.

49. Hackel MA, Tsuji M, Yamano Y, et al. In vitro activity of the siderophore cephalosporin, cefiderocol, against carbapenem-nonsusceptible and multidrug-resistant isolates of Gram-negative bacilli collected worldwide in 2014 to 2016. Antimicrob Agents Chemother 2018;62:e1968-17.

50. Portsmouth S, Vennhuyzen D, Echols $\mathrm{R}$, et al. Cefiderocol compared with imipenem/cilastatin in the treatment of adults with complicated urinary tract infections with or without pyelonephritis or acute uncomplicated pyelonephritis: results from a multicentre, doubleblind, randomized study. 2017 Presented at 27th European Congress of Clinical Microbiology and Infectious Diseases, Vienna.

51. Shionogi, Inc. 2016. Study of S-649266 or best available therapy for the treatment of severe infections caused by carbapenem-resistant Gram-negative pathogens (CREDIBLE-CR). Accession no. NCT02714595. ClinicalTrials.gov, NIH. Available from: https://clinicaltrials.gov/ct2/ show/NCT02714595

52. Shionogi, Inc. 2017. Clinical study of S-649266 for the treatment of nosocomial pneumonia caused by Gram-negative pathogens (APEKS-NP). Accession no. NCT03032380. ClinicalTrials.gov, NIH. Available from: https://clinicaltrials.gov/ ct2/show/NCT03032380

53. Thye D. GT-1: A novel siderophore cephalosporin for MDR Gram-negative pathogens - as monotherapy and in combination with GT-055, a novel betalactamase inhibitor new agents discovery summary session: early new antimicrobial agents. Presented at ASM Microbe, 2018. Available at: https://www.ihma.com/app/uploads/Ge o m P 40 G T - 1 A S M 2018_FINAL.pdf. Accessed on: 27/05/ 2019

54. Wehrli W, Zimmermann W, Kump W, et al. CGP 4832, a new semisynthetic rifamycin derivative highly active against some Gram-negative bacteria. J Antibiot 1987;40:1733-9.

55. Pugsley AP, Zimmerman W, Wehrli W. Highly efficient uptake of a rifamycin derivative via the FhuATonBdependent uptake route in Escherichia coli. J Gen Microbiol 1987;133:3505-11.

56. Wencewicz TA, Long TE, Möllmann U, 
et al. Trihydroxamate siderophore- fluoroquinolone conjugates are selective sideromycin antibiotics that target Staphylococcus aureus. Bioconjug Chem 2013;24:473-86.

57. Ghosh M, Lin YM, Miller PA, et al. Siderophore conjugates of daptomycin are potent inhibitors of carbapenem resistant strains of Acinetobacter baumannii. ACS Infect Dis 2018;4:152935.

58. Sritharan M. Iron homeostasis in Mycobacterium tuberculosis: mechanistic insights into siderophore-mediated iron uptake. J Bacteriol
2016;198:2399-409.

59. Patel K, Butala S, Khan T, et al. Mycobacterial siderophore: a review on chemistry and biology of siderophore and its potential as a target for tuberculosis. Eur J Med Chem 2018;157:78390.

60. Hider RC, Kong X. Chemistry and biology of siderophores. Nat Prod Rep 2010;27:637-57.

61. Nagoba B, Vedpathak D. Medical applications of siderophores. Eur J Gen Med 2011;8:229-35.

62. Saha M, Sarkar S, Sarkar B, et al. Microbial siderophores and their poten- tial applications: a review. Environ Sci Pollut Res Int 2016;23:3984-99.

63. Rümenapp C, Gleich B, Haase A. Magnetic nanoparticles in magnetic resonance imaging and diagnostics. Pharm Res 2012;29:1165-79.

64. Kaeopookum P, Summer D, Pfister J, et al. Modifying the siderophore triacetylfusarinine $\mathrm{C}$ for molecular imaging of fungal infection. Mol Imaging Biol 2019 [Epub ahead of print].

65. Petrik M, Zhai C, Haas $H$, et al. Siderophores for molecular imaging applications. Clin Transl Imaging 2017;5:15-27. 